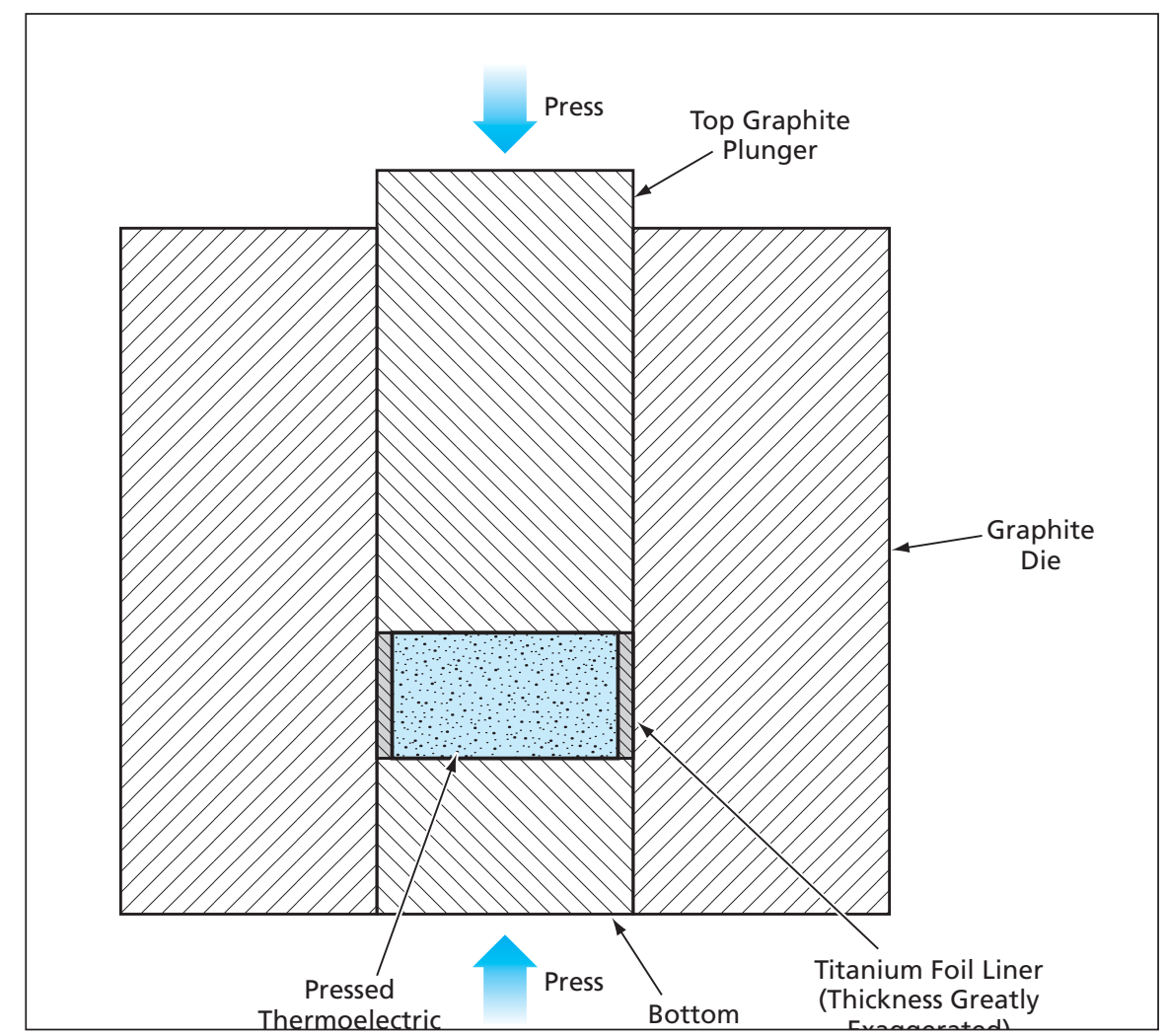

In a Powder-Metallurgy Process used to fabricate a segment of a thermoelectric device, the particles of thermoelectric material are sintered by heat and pressure, which is also exploited to bond the sintered mass to the outer layer of titanium foil.

Guided by the aforementioned experiments, a powder-metallurgy process for fabricating skutterudite was modified to provide for covering the outer surfaces of the segments with titanium foils. In the unmodified process, the thermoelectric material, in powder form, is hotpressed in a graphite die, then removed, then further processed. The combination of high temperature and pressure in the die acts to promote bonding between particles, and as such, is ideal as a means of adding an adherent sublimation-suppressing outer layer. Hence, the process is modified by simply lining the inner wall of the die with a foil of the barrier material before filling the die with the thermoelectric powder (see figure).

In preparation for further experiments, the modified process was used to fabricate specimens of $\mathrm{n}$ - and p-type skutterudites covered with adherent 25$\mu \mathrm{m}$-thick foils of titanium. In the experiments, these specimens were heated in a vacuum under the same conditions as in the experiments described above, then sectioned and examined. Like the niobium foils in those experiments, the titanium foil outer layers in these experiments were found to have suppressed sublimation of $\mathrm{Sb}$.

This work was done by Jeffrey Sakamoto, Thierry Caillat, Jean-Pierre Fleurial, and G. Jeffrey Snyder of Caltech for NASA's Jet Propulsion Laboratory. Further information is contained in a TSP (see page 1).

This invention is owned by NASA, and a patent application has been filed. Inquiries concerning nonexclusive or exclusive license for its commercial development should be addressed to the Patent Counsel, NASA Management Office-JPL. Refer to NPO-40040.

\title{
SUltrahigh-Temperature Ceramics
}

\section{Materials are being developed to withstand temperatures above $1,650{ }^{\circ} \mathrm{C}$.}

\section{Ames Research Center, Moffett Field, California}

Ultrahigh temperature ceramics (UHTCs) are a class of materials that include the diborides of metals such as hafnium and zirconium. The materials are of interest to NASA for their potential utility as sharp leading edges for hypersonic vehicles. Such an application requires that the materials be capable of operating at temperatures, often in excess of $2,000{ }^{\circ} \mathrm{C}$. UHTCs are highly refractory and have high thermal conductivity, an advantage for this application. UHTCs are potentially applicable for other high-temperature processing applications, such as crucibles for molten-metal processing and high-temperature electrodes.

UHTCs were first studied in the 1960 's by the U.S. Air Force. NASA's Ames Research Center concentrated on developing materials in the $\mathrm{HfB}_{2} / \mathrm{SiC}$ family for a leading-edge application. The work focused on developing a process to make uniform monolithic (2phase) materials, and on the testing and design of these materials. Figure 1 shows arc-jet models made from UHTC materials fabricated at Ames. Figure 2 shows a cone being tested in the arc-jet. Other variations of these materials being investigated elsewhere include zirconium-based materials and fiber-reinforced composites.

Current UHTC work at Ames covers four broad topics: monoliths, coatings, composites, and processing. The goals include improving the fracture toughness, thermal conductivity and oxidation resistance of monolithic UHTCs and developing oxidation-resistant UHTC coatings for thermal-protection-system sub-

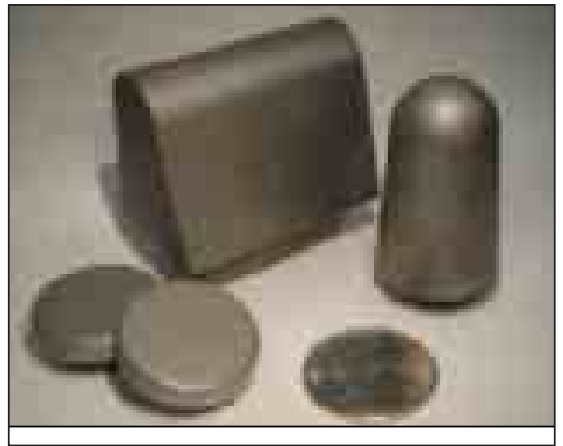

strates through novel coating methods.

Figure 1. Examples of UHTC Components are shown that have been tested in the NASA Ames Arc Jet facility to evaluate the materials response in a simulated reentry environment. The cone and wedge models are representative of the scale and geometries anticipated for use on UHTC sharp leading-edge vehicles. 


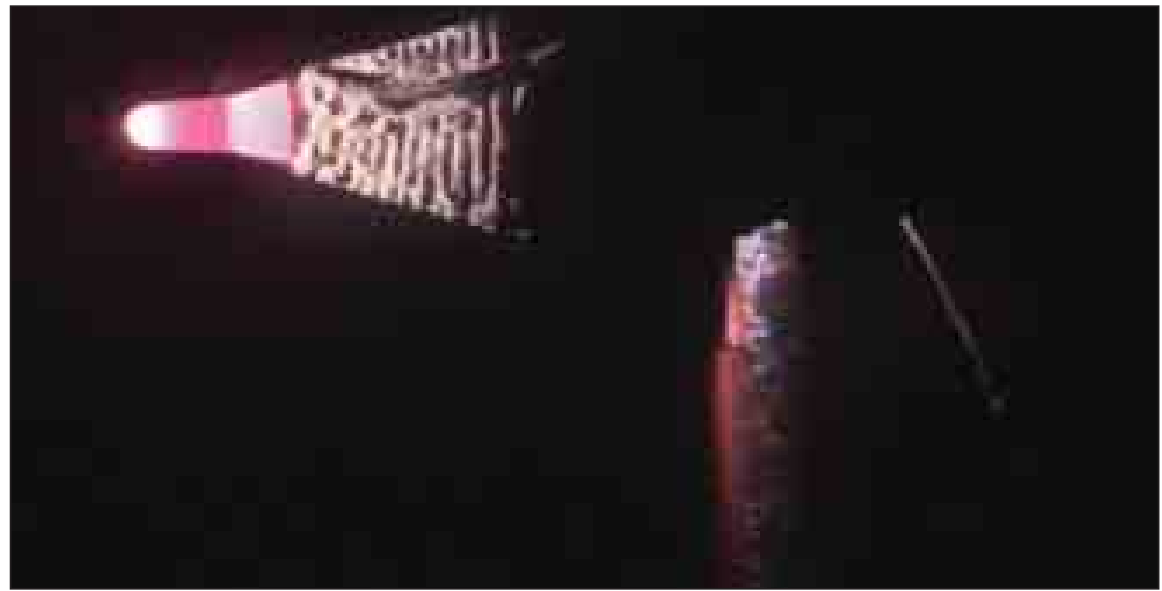

Figure 2. A UHTC Cone is shown during testing in the NASA Ames Arc Jet facility. Surface temperatures on the tip of the cone model exceeded $2,000^{\circ} \mathrm{C}$ during this test.
As part of this effort, researchers are exploring compositions and processing changes that have yielded improvements in properties. Computational materials science and nanotechnology are being explored as approaches to reduce materials development time and improve and tailor properties.

This work was done by Sylvia M. Johnson, Donald T. Ellerby, Sarah E. Beckman, and Edward Irby of Ames Research Center and Matthew J. Gasch and Michael I. Gusman of ELORET. Further information is contained in a TSP (see page 1).

Inquiries concerning rights for the commercial use of this invention should be addressed to the Ames Technology Partnerships Division at (650) 604-2954. Refer to ARC-15258-1.

\section{Improved C/SiC Ceramic Composites Made Using PIP}

These materials are expected to remain strong for longer times at high temperatures.

\section{Marshall Space Flight Center, Alabama}

Improved carbon-fiber-reinforced $\mathrm{SiC}$ ceramic-matrix composite $(\mathrm{C} / \mathrm{SiC} \mathrm{CMC})$ materials, suitable for fabrication of thick-section structural components, are producible by use of a combination of raw materials and processing conditions different from such combinations used in the prior art. In comparison with prior $\mathrm{C} / \mathrm{SiC} \mathrm{CMC}$ materials, these materials have more nearly uniform density, less porosity, and greater strength. The majority of raw-material/processing-condition combinations used in the prior art involve the use of chemical vapor infiltration (CVI) for densifying the matrix.

In contrast, in synthesizing a material of the present type, one uses a combination of infiltration with, and pyrolysis of, a preceramic polymer [polymer infiltration followed by pyrolysis (PIP)]. PIP processing is performed in repeated, tailored cycles of infiltration followed by pyrolysis. Densification by PIP processing takes less time and costs less than does densification by CVI. When one of these improved materials was tested by exposure to a high-temperature, inert-gas environment that caused prior $\mathrm{C} / \mathrm{SiC} \mathrm{CMCs}$ to lose strength, this material did not lose strength. (Information on the temperature and exposure time was not available at the time of writing this article.)

A material of the present improved type consists, more specifically, of (1) carbon fibers coated with an engineered fiber/matrix interface material and (2) a ceramic matrix, containing $\mathrm{SiC}$, derived from a pre-ceramic polymer with ceramic powder additions. The enhancements of properties of these materials relative to those of prior $\mathrm{C} / \mathrm{SiC}$ CMC materials are attributable largely to engineering of the fiber/ matrix interfacial material and the densification process.

The synthesis of a material of this type includes processing at an elevated temperature to a low level of open porosity. The approach followed in this processing allows one to fabricate not only simple plates but also more complexly shaped parts. The carbon fiber reinforcement in a material of this type can be in any of several alternative forms, including tow, fabric, or complex preforms containing fibers oriented in multiple directions.

This work was done by Timothy Easler of COI Ceramics Inc. (an Affiliate of ATK Space Systems) for Marshall Space Flight Center. Further information is contained in a TSP (see page 1). . Refer to MFS-32384-1.

\section{Coating Carbon Fibers With Platinum}

\section{Uniform coats are produced relatively inexpensively.}

\section{Marshall Space Flight Center, Alabama}

A process for coating carbon fibers with platinum has been developed. The process may also be adaptable to coating carbon fibers with other noble and refractory metals, including rhenium and iridium. The coated carbon fibers would be used as ingredients of matrix/fiber composite materials that would resist ox- idation at high temperatures. The metal coats would contribute to oxidation resistance by keeping atmospheric oxygen away from fibers when cracks form in the matrices.

Other processes that have been used to coat carbon fibers with metals have significant disadvantages:
- Metal-vapor deposition processes yield coats that are nonuniform along both the lengths and the circumferences of the fibers.

- The electrical resistivities of carbon fibers are too high to be compatible with electrolytic processes.

- Metal/organic vapor deposition en- 